\title{
A measure of trade induced adjustment in volume and quality space.
}

\begin{abstract}
In this paper we contribute to the literature on the measurement of international trade flows. Specifically, we combine changes in the volume and quality in matched trade changes to present a simple new index together with a geometric framework that can be used to visualise changes in quality and volume simultaneously. We illustrate the usefulness of our simple extension with data for trade between Malaysia and China between 1994 and 2004.
\end{abstract}

Keyword: Exports; Intra-industry trade; Adjustment costs; Quality; Product differentiation. 